\title{
Forma topológica y geométrica, función proyectiva y operativa, y la estructura de los objetos
} Topological and geometric form, projective and operational function, and the structure of objects

\section{Mauricio Ordóñez-Chacón ${ }^{1}$}

Ordoñez-Chacón, M. Forma topológica y geométrica, función proyectiva y operativa, y la estructura de los objetos. Tecnología en Marcha. Edición especial. Escuela de Arquitectura y Urbanismo, Diciembre 2020. Pág 118-130.

doi) https://doi.org/10.18845/tm.v33i8.5514 


\title{
Palabras clave
}

Forma geométrica; forma topológica; función; estructura; necesidad; teoría de la arquitectura.

\section{Resumen}

Ensayo investigativo teórico con finalidad didáctica y profesional. Se concentra en los conceptos de forma, función y estructura como categorías analíticas para la teoría de los objetos artificiales funcionales, con algunas precisiones en el ámbito de la arquitectura. Aparte de aclarar conceptos para la enseñanza teórica, es una epistemología instrumental para el proceso de diseño. Entre más claridad de estas categorías, más preciso el diseño como práctica tecnológica blanda dirigida a la modificación entorno. Por tratarse de una teoría fundamentadora, los conceptos provienen de bibliografía clásica, no efímera, y de diccionarios filosofía y lengua. Bajo el método empírico-analítico se cotejan conceptos con una profunda reflexión analítica tras la relación experimental con los objetos artificiales y sus propiedades. Abundante literatura sobre el diseño definen y utilizan estos conceptos, muchas veces sin profundizar en ellos o de manera contradictoria. Ha existido ambigüedad en el concepto de forma, además de sufrir una cuestionable contaminación estética; se enfatiza en su carácter morfológico y se proponen como suficientes las categorías de forma geométrica y forma topológica. El concepto de función, reducido a la utilidad práctica de los objetos, se amplía a su función semiótica. El concepto de estructura sistémica, ha sufrido una deformación conceptual al reducirse a la estructura física soportante de los objetos. Se plantea corregir estas desviaciones conceptuales, y enriquecer con sub-categorías.

\section{Keywords}

Geometrical form; topological form; function; structure; necessity; theory of architecture.

\begin{abstract}
Theoretical research essay for educational and professional purposes. It concentrates on the concepts of form, function and structure as analytical categories for the theory of functional artificial objects, with some clarifications in the field of architecture. Apart from clarifying concepts for theoretical teaching, it is an instrumental epistemology for the design process. The more clarity of these categories, the more precise the design as a soft technological practice aimed at environment modification. As it is a founding theory, the concepts come from classical bibliography, not ephemeral, and from philosophy and language dictionaries. Under the empirical-analytical method concepts are collated with a deep analytical reflection after the experimental relationship with artificial objects and their properties. These concepts are defined and used in abundant design literature, many times without going into them or in a contradictory way. There has been ambiguity in the concept of form, in addition to suffering a questionable aesthetic contamination; its morphological character is emphasized and the categories of geometric shape and topological shape are proposed as sufficient. The concept of function, reduced to the practical utility of objects, is extended to its semiotic function. The concept of systemic structure has suffered a conceptual deformation as it has been reduced to the supporting physical structure of objects. It is proposed to correct these conceptual deviations, and enrich with sub-categories.
\end{abstract}




\section{Introducción}

Fundamentalmente se exploran los conceptos de necesidad, forma, función y estructura como categorías analíticas para la comprensión de los objetos artificiales funcionales en su contexto. Dentro del ámbito del diseño, existe abundante literatura al respecto, pero una diversidad de interpretaciones. La tríada forma-función-estructura, que tiene un origen en la ciencias sociales y políticas, ha sido empleada en el diseño con varias distorsiones conceptuales, tal como se demostrará, además de no ser lo suficientemente detalladas y comprehensivas para una analítica del fenómeno del objeto diseñado y su contexto, por lo cual se agregan otras subcategorías.

\section{Desarrollo}

\section{Forma}

El término 'forma' es uno de los más elusivos y multívocos dentro de la teoría arquitectónica y del diseño. Una de las razones es porque ha tomado conceptos de diversas disciplinas como la filosofía, matemática, artes, ciencias básicas, sicología (gestalt), etc, no siempre aplicables al problema de los objetos diseñados. Ya en el s.XII, Gilbert de la Porée corroboraba que "se habla de la forma en muchos sentidos" [1, p.276]. Forma, en latín, se traduce como forma; hermosura; molde y horma; imagen y figura; tipo; configuración y constitución. En Historia de Seis Ideas, Tatarkiewicz señala: "Desde el principio, el término latino 'forma' sustituyó dos palabras griegas: morfh y eidoz (morfé y eidós), la primera aplicada a las formas físicas, la segunda a las formas conceptuales. Esta doble herencia ha contribuido considerablemente a la diversidad de significados que tiene el término forma" [1, p.253]. Una dualidad bastante semejante a la "res extensa" y "res cogitans" de Descartes. Y parece que esta doble raíz es la que genera las dicotomías forma-materia y forma-contenido, tal como se manejan en diversas disciplinas donde, en el primer caso, la forma sería el eidós que organiza la morfé, y en segundo caso lo contrario, la forma es morfé que comunica eidós.

Históricamente, diversas acepciones de forma: -La disposición de las partes, su ordenamiento y composición, con su propia topología, una eidós que organiza la morfé. - La apariencia sensorial de las cosas, contrario a su contenido o mensaje, la materia configurada y sensible que comunica ideas, morfé que comunica eidós. Para Hegel, la existencia empírico-concreta del fenómeno. -El contorno de las cosas, propiedad superficial percibida de la misma materia sensible, derivado de su geometría. -La forma substancial de Aristóteles, que le concede a la forma un poder ontológico creador, pues al configurar la materia determina que "sea algo" y no otra cosa, siendo la relación entre materia y forma similar a la de potencia y acto. -La forma kantiana, más epistemológica, que corresponde a "estructuras mentales que hacen posible ordenar el material de la experiencia" [2], concepto incorporado en la teoría de la Gestalt, donde la forma es el reconocimiento de una estructura organizacional entre una serie de elementos percibidos sensorialmente, para delimitarlo como conjunto distinguible. -La forma lógica, estructura conceptual que trabaja las "proposiciones formales, independientes de los hechos factuales", ciertamente más topológica y estructural [3].

¿Cuál sería la definición idónea de forma que se encuentra en los objetos o artefactos físicos artificiales, diseñados y construidos por el ser humano? En su aspecto ontológico, con consecuencias prácticas, especialmente apta sería la definición aristotélica de forma, pues en ella encontramos una forma que organiza la materia para que 'sea algo', o sea, la forma es la estructura organizadora de la materia, el modo como se organiza la materia, y al hacerlo define la naturaleza o esencia del objeto, la categoría o clase de objeto, el qué es, su ontología. 
Bajo ese principio, y con finalidad práctica morfogenética, se proponen la forma geométrica y la forma topológica como definitivas para la morfología de las cosas. Estas formas son abstractas, pero son las que organizan la materia. La forma geométrica define la configuración de la masa material, la apariencia sensorial de su contorno, la que toma la materia para que sea algo y no otra cosa. La forma topológica es la forma lógica estructural que organiza la disposición o distribución de las partes, independientemente de su geometría y materialidad. La materia se configura por la interrelación de estas dos formas implícitas fundamentales.

La forma geométrica es la base para configurar (dar forma, moldear) la materia y el espacio físico (ver figura 1). Toda materia se organiza por medio de una geometría implícita. La forma geométrica pura es abstracta, no tiene escala ni materia ni funciones, lo cual marca la diferencia con la forma arquitectónica, pero es utilizada por ésta -y por el diseño en general- para facilitar el pensamiento y la sintaxis de la formas materiales-espaciales, tanto en su diseño, representación y construcción ${ }^{2}$. La escala, proporción y modulación son relaciones geométricas típicas.

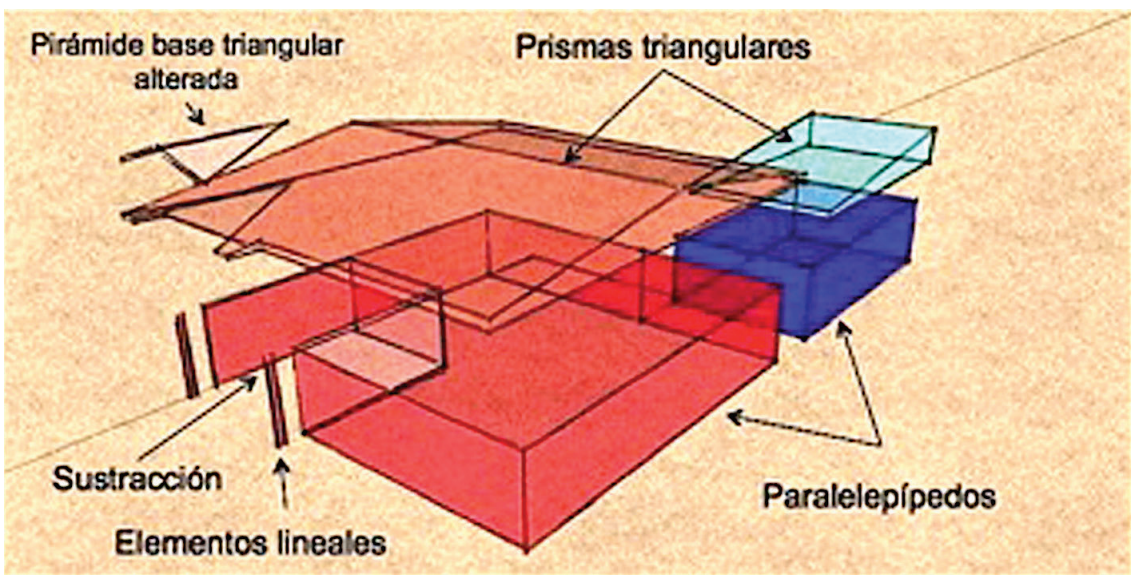

Figura 1. Forma geométrica de una vivienda.

La forma topológica todavía es más abstracta, es una estructura lógica (formal) independiente de las características geométricas y materiales (fácticas) de sus componentes. En ella, los componentes físicos son simples entidades en las cuales se analizan las relaciones invariantes pese a los cambios geométricos o materiales de los elementos. De manera simplificada y aplicada a problemas espaciales, nos referimos al borde o cerramiento que generan la existencia o no de entidades espaciales, a las relaciones de proximidad, contigüidad (adyacencia y accesibilidad), transitividad, continuidad (como contigüidad de similitudes), intersección e inclusión entre ellas, que producen estructuras espaciales lineales, en árbol o tramadas según como se organizan nodos y conectores (entidades y relaciones). La accesibilidad (conectividad) o simple adyacencia (muros entre espacios contiguos) son relaciones topológicas a tomar en cuenta por sus potencialidades prácticas arquitectónicas (ver figura 2). ${ }^{3}$

2 Una profundización sobre la diferencia entre forma geométrica y forma arquitectónica puede encontrarse en Boudon [4, pp.50-60].

3 Antecedente de esta visión topológica en el urbanismo es el famoso artículo "La Ciudad no es un árbol", de Christopher Alexander [5]. Documentación inteligible sobre la topología en arquitectura la podemos encontrar en Millán [6] y en un artículo didáctico de Blanco y de Prada [7], entre otras. 


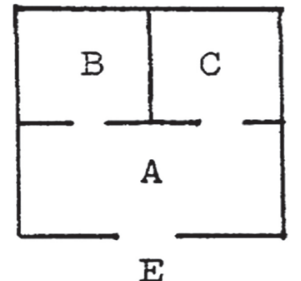

$E$

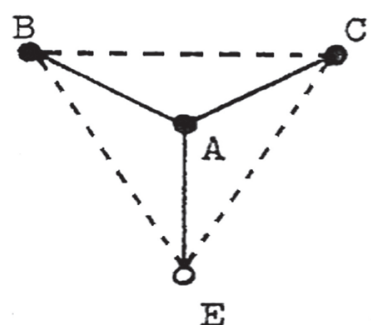

E

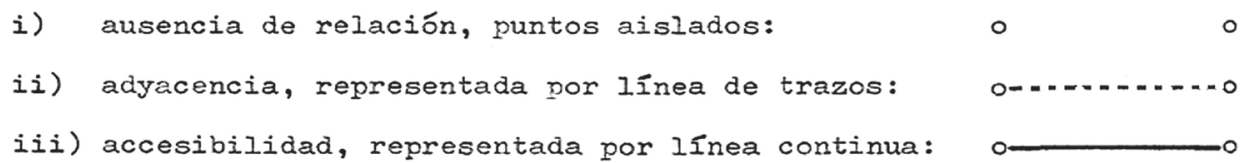

Figura 2. Relaciones de adyacencia y accesibilidad. Fuente: [6, p.149]

La forma topológica es estructura pura y profunda en el sentido estructuralista del término, pues se concentra en las relaciones, independientemente de las entidades en juego. Por ejemplo, no importa si el baño es cilíndrico o rosado, lo que importa en topología es que sea una entidad espacial y esté contiguo al dormitorio; o, tratándose de una cruz, lo topológico es que sus componentes se intersecan, independientemente de su geometría, ángulo, dimensión o materia. La forma topológica es forma lógica, puramente formal en el sentido matemático del término. Es la estructura gramática que organiza la sintaxis de las distintos componentes de un objeto. Podemos ver en una simple mesa las formas geométrica y topológica interactuando (ver figura 3).

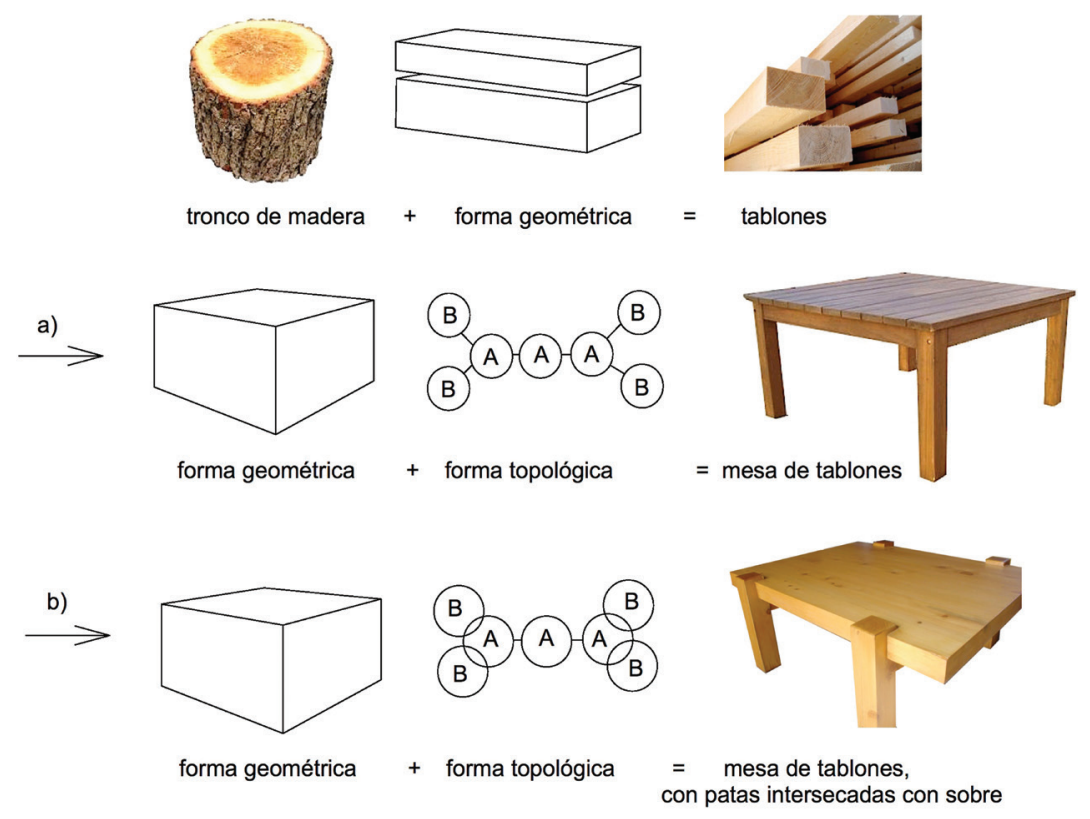

Figura 3. Forma geométrica y forma topológica en una mesa.

De igual manera, los distintos materiales de una obra arquitectónica son configurados y ensamblados. Y la composición espacial o distribución de los espacios arquitectónicos es otra configuración topológica. Para ilustrar cómo una misma topología puede tener distintas geometrías, los planos de casas de F.L.Wright se muestran en la figura 4. 


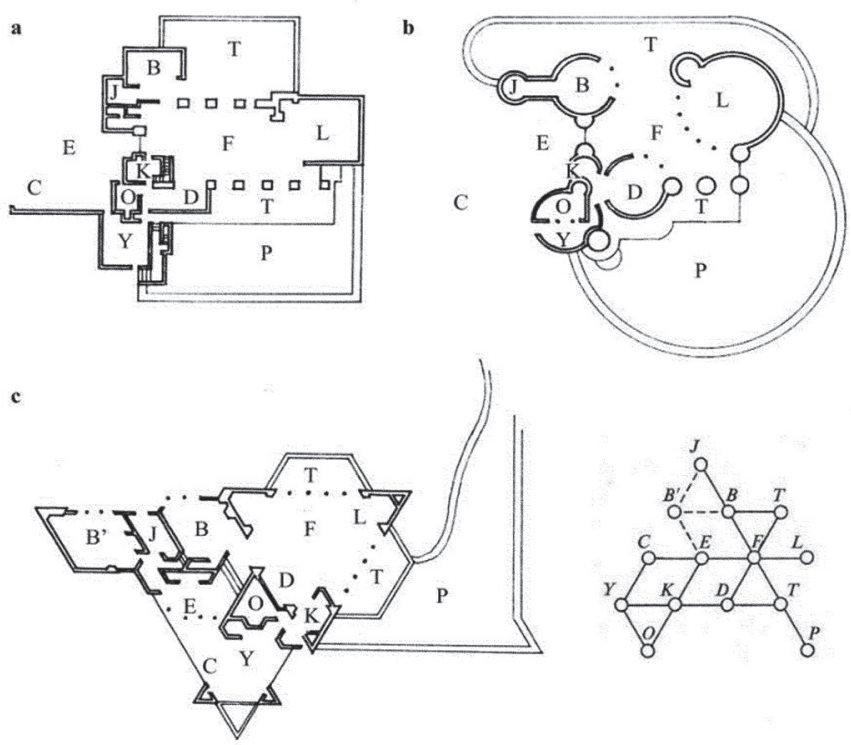

Figura 4. Planos de casas de F.L.Wright, por March y Steadman, 1971. Fuente: [6, p.142].

La distribución espacial puede observarse mejor por medio de diagramas topológicos, donde se combinan grafos y diagramas de Euler propios de la teoría de conjuntos (ver figura 5).

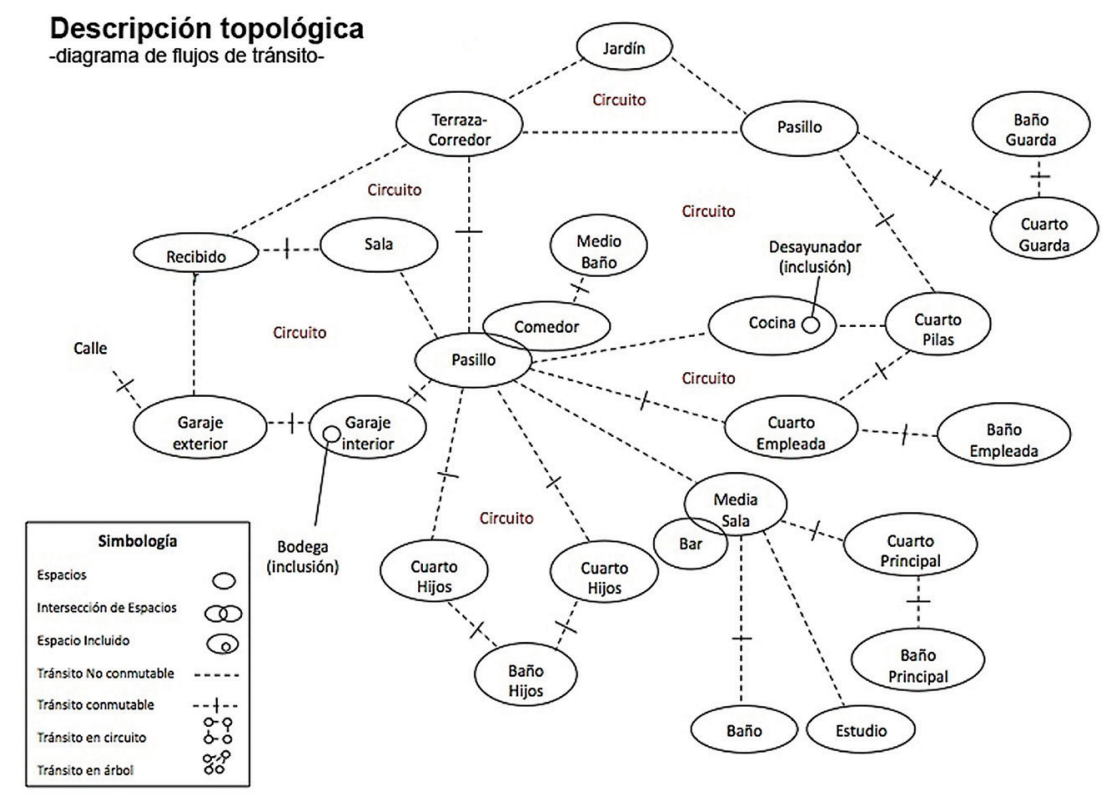

Figura 5. Diagrama topológico espacial.

Es importante, a la hora de analizar o diseñar una forma arquitectónica o urbana, hacer diagramas topológicos y síntesis geométricas de los componentes estudiados. Esta lectura "desplumada", limpia de materialidad y detalles secundarios, permite ver la estructura organizativa pura de una organización espacial, y analizar ciertas cualidades utilitarias, semióticas o de estabilidad con mayor claridad. Por ejemplo, la proporción, como característica geométrica, no solo se presta para valoraciones estéticas, sino de utilidad o estabilidad. 
Pero es epistemológicamente relevante distinguir entre la forma en sí, y las cualidades que posee, según la intención deseada. Cuando hablamos de una estructura formal, se trata de una morfología que únicamente se describe, un sustantivo, dejando fuera cualquier connotación estética, por ejemplo, cualidad que correspondería a la hipestructura funcional de esa forma, parte de sus cualidades semióticas. Corresponde aquí denunciar la contaminación estética del concepto de forma: Históricamente, según Tatarkiewicz, el concepto de forma A no aludía a cualquier disposición y proporción de las partes, sino aquella que por su orden, medida y proporción produce belleza. Para los griegos, y después para los latinos, la palabra forma implicaba automáticamente belleza: "El adjetivo 'formosus' (hermoso) fue incorporado bastante pronto al lenguaje del arte. Este derivado de forma significaba que tenía una buena proporción, que era bella, que sugería un juicio estético favorable... Lo mismo sucedió con el adjetivo negativo 'deformis' (deformidad y fealdad)". Y Clarembaldus de Arras decía: "La forma es la disposición adecuada en las cosas materiales" [1, p.258], es decir, no cualquier forma.

El mismo Vitruvio tiende a esta mezcla o confusión conceptual entre forma y belleza: Si en su Libro I hablaba de 'venustatis' (genitivo de 'venustas') para referirse a la belleza o atractivo, en su Libro III utiliza el término 'ad speciem' para lo mismo, que viene de 'species', que significa el aspecto, forma o manifestación exterior de una cosa, aunque también se aplicaba para el aspecto bello o belleza. Sin embargo, al final del mismo párrafo aclara que lo que se busca no es cualquier aspecto, sino un aspecto bello, utilizando nuevamente el adjetivo 'venustum' [8].

Quizá por aquí anda el origen de la contaminación estética del concepto de forma, pues no se aplica a cualquier disposición u ordenamiento, hasta los realizados por razones de utilidad, estabilidad o economía, sino casi a los ordenamientos con afán estético, o al análisis estético de estos ordenamientos. Se confunde la 'species' con la 'venustas', la forma con la buena forma, la forma-sustantivo con un adjetivo cualitativo de la forma., la morfología con la estética.

Varios han enfatizado el exclusivo carácter morfológico de la forma: Henri Lefebvre cuando dice, hablando de la forma, que "el examen de los efectos estéticos o "efectos de significado" no tiene ningún derecho de prioridad en este contexto" [9, p.198]. Norberg-Schulz [10]: "La dimensión formal es independiente de las dimensiones semántica y pragmática", refiriéndose a los significados (estéticos, simbólicos, etc) y el pragmatismo de lo utilitario. Tedeschi [11]: “... es la forma como hecho objetivo, independientemente de lo que pueda ser su valor como expresión o símbolo, su significado a momentos de gusto". Borie y et al [12, p.34]: "Al ser nuestro objetivo revelar la coherencia interna de las formas urbanas o arquitectónicas, es necesario fijar unos criterios exclusivamente morfológicos que, por supuesto, se podrán poner luego en relación con cualquier otro tipo de criterio, sea funcional, semiótico o perceptivo". ${ }^{4}$

Pero esto no cala todavía en la teoría del diseño. Lo que se denomina formalismo en el arte plástico, e incluso en la arquitectura, generalmente alude a este énfasis en lo estético y lo plástico, o de otra índole semiótica, por sobre consideraciones prácticas utilitarias. Este afán formalista-expresionista, en arquitectura, a menudo deviene en un diseño de formas expresivas a priori donde luego se busca cómo encajar las actividades para que el edificio cumpla con su función utilitaria, contrario a la actitud funcionalista donde la forma surgiría de adentro hacia fuera, como una resultante de las cualidades prácticas buscadas [13]. Cierto formalismo puede ser también por razones económicas (el clásico "cajón" más rápido, estable y barato). Pero en rigor al término, todos los diseñadores son "formalistas", pues todos manipulan y trabajan

4 De acuerdo con Borie y compañeros, excepto en el deslinde que hacen respecto a lo perceptivo. La percepción es un acto fisiológico-cognitivo esencial para tener contacto con cualquier forma, para denotarla y connotarla, para poder entenderla y usarla tanto utilitaria como semióticamente. La percepción es fundamental para el análisis morfológico, que de otra manera no sería posible. 
formas; hasta el ingeniero más desinteresado en la estética igual manipula la forma de la materia con otras intenciones. Si hay especial afán estético, sería más más apropiado llamarlo "esteticismo", y "expresionismo" si se quieren expresar otras intenciones semióticas.

\section{Estructura}

Bajo la concepción lógica y gestáltica, forma y estructura, en su sentido organizacional, se manejan casi como sinónimos: la forma es una estructura, y toda estructura es (tiene) una forma. "En la arquitectura, forma y estructura no son más que un solo y un mismo fenómeno..." dicen Borie et al [12, p.27]. Los filósofos soviéticos Rosental y Straks [14] señalan: "Cada organismo posee determinada estructura, una cierta organización de los elementos materiales que lo integran, es decir, una forma... La forma es la organización, la estructuración del contenido". Especialmente estructural es la forma topológica. Pero no conviene confundir ambos conceptos.

La estructura es el orden o modo de ser de los sistemas u organizaciones que se refiere fundamentalmente a las relaciones o vínculos entre las partes. Distribución y orden de las partes de una cosa, cuerpo, obra o edificio, dice el diccionario. "Las interrelaciones más o menos estables entre las partes o componentes de un sistema, que pueden ser verificadas (identificadas) en un momento dado, constituyen la estructura del sistema... En algunos casos es preferible distinguir entre una estructura primaria (relaciones internas) y una hiperestructura (relaciones externas)" [15]. "Al hablar de estructura hacemos a un lado, abstraemos sus elementos, y consideramos solo las correlaciones importantes. Sin incluimos además a los elementos -actuando unos sobre otros- obtenemos el sistema" [16, p.91]. "Esa es la tarea del análisis estructural, relativa a los vínculos entre el todo y las partes, lo macro y lo micro" [9, p.207]. Este es el sentido 'estructuralista' y sistémico del término. Los vínculos o relaciones estructurales o sistémicos pueden ser de materia, energía o información, o combinados. Y toda estructura tiene una forma, o modo de organizarse.

Hay que distinguir las diversas estructuras en un objeto: La estructura física de su materialidad, estructura primaria en la configuración y ensamblaje de sus partes, lograda por las técnicas y tecnologías constructivas; con su implícita estructura formal en la geometría de sus partes y del todo, y en la forma topológica que las relaciona (contigüidad lineal-en árbol-en trama, intersección, inclusión, etc). Una estructura funcional interna entre las partes del objeto (por ej: la estructura soportante sostiene las envolventes), y otra estructura funcional externa entre el objeto y el usuario, tanto a nivel utilitario como semiótico. Y una estructura del contexto, también externa o hiperestructural, de los entornos entre sí (humano, construido y natural) y cómo afectan al objeto con parámetros contextuales en lo social, cultural, la economía, ecología, arte, ciencia, técnica y tecnología, hasta llegar a las llamadas superestructuras como la ideología, política y leyes. La forma y la función tienen sus estructuras.

Por ello hay que tener cuidado, especialmente en arquitectura, con el uso de la palabra estructura a secas, puesto que estructuras encontramos en todo sistema organizado, tanto macrofísico como conceptual. Al menos en la literatura arquitectónica, el término ha tendido a confundirse con el de estructura soportante (marcos estructurales, planos rígidos, etc), que es un componente físico de las edificaciones o de otros artefactos construidos, o de los objetos y seres naturales (esqueleto), atroz confusión en la que cayeron Nervi y muchos más, como se verá más adelante.

\section{Función (y necesidad)}

¿Cuál es la razón de ser, finalidad o teleología que lleva a la creación de los artefactos u objetos materiales artificiales? Satisfacer ciertas necesidades humanas. Por necesidad se puede entender "la dependencia del ser viviente, en cuanto a su vida o sus intereses, de otras 
cosas o seres" [17, p.756], donde cita la carencia y la perturbación de cierto equilibrio orgánico que mueve a su restablecimiento. Sistémicamente, la necesidad implicaría la demanda de recursos o la modificación del entorno para mantener la homeostasis (preservación) o lograr determinada morfogénesis (transformación). Bronisław Malinowski [18] desarrolló su teoría de las necesidades humanas, algunas fundamentales para la sobrevivencia: nutrición, reproducción, seguridad, relajación, crecimiento, aprendizaje, etc. Abraham Maslow [19] creó su jerarquía de necesidades, desde las más básicas o fisiológicas hasta las más simbólicas y emocionales, que junto con las estéticas, igualmente entran dentro de las categoría de necesidades.

La finalidad de los objetos físicos artificiales es diversa, según las necesidades a satisfacer, en todas las ramas del diseño: utensilios, ropa, muebles, arquitectura, urbanismo, etc. Respecto a la arquitectura y el urbanismo, la finalidad es proveer un espacio habitable, en el sentido más amplio del término. Hablamos de una relación entre el objeto diseñado y el entorno humano que lo demanda o lo percibe (usuario), ya sea por razón utilitaria o semiótica, quien lo usa en su práctica social. (Generalmente sinónimos entorno y contexto, aunque se sugiere que el entorno sea esa parte del contexto modificada por el objeto). Dentro de lo semiótico se incluyen aquí las finalidades estéticas, simbólicas, ideológicas e incluso emocionales.

Y siempre, el entorno humano se encuentra rodeado por los entornos construido y el entorno natural. Son las condiciones extrínsecas que actúan sobre la forma del objeto. La forma busca ajustarse a las necesidades del entorno humano específico, pero mediatizadas por los características de su contexto. El contexto opera como parámetro para el objeto, y su forma y materialidad es la variable, la que tiene que ajustarse en busca de equilibrio. Aunque el objeto también produce cambios en el contexto (causa-efecto). Una estructura sistémica.

Diáfanos y poderosos son estos párrafos de Christopher Alexander: "Todo problema de diseño se inicia con un esfuerzo de lograr un ajuste entre dos entidades: la forma en cuestión y su contexto. La forma es la solución para el problema; el contexto define el problema" [20, p.21]. Y afina: "La forma es esa parte del mundo que está bajo nuestro control y que decidimos modelar en tanto que dejamos el resto del mundo tal cual. El contexto aquella parte del mundo que hace exigencias a la forma... El ajuste es una relación de mutua aceptabilidad entre estos dos elementos" [19, p.24]. Ahonda en sus notas que este ajuste es una adaptación tanto de la forma a su contexto como del contexto a la forma, similar al concepto de adaptación darwiniana entre organismo y medio ambiente. Nótese que incluye al usuario dentro del contexto, usa el término forma comprehensivamente, incluyendo la materia por ella configurada.

Las mismas categorías analíticas para evaluar ese ajuste entre forma y contexto -utilidad práctica, semiótica, estabilidad y costo económico y ambiental, materia de otro ensayo- pueden servir para investigar las necesidades humanas en su entorno. Hay una relación biunívoca entre las cualidades del objeto diseñado y las necesidades o deseos humanos que satisfacen.

La función es la capacidad práctica del objeto para cumplir o realizar su finalidad o propósito, aunque puede cumplir otras funciones no previstas. El objeto funciona si se ajusta en buen grado a las demandas y necesidades del usuario; si no lo logra, se dice que el objeto no funciona, o funciona mal. Este ajuste entre objeto y contexto es tarea del diseño, pero ese ajuste solo se verifica durante la praxis funcional, una relación práctica operativa entre el objeto y el entorno humano que lo usa. La función es una cualidad relacional, una relación hiperestructural. La función no está en el objeto, sino en su relación con el usuario.

En el estadio teórico de diseño, la función proyectiva consistiría en la activación operativa hipotética de ese objeto, y cumple una labor morfogenética sobre la forma: una forma inicial se transforma en otra por efecto de la función prevista en el ámbito funcional (utilidad, significados) o por otra razones (estabilidad, costos). Esta función transformadora es semejante al concepto matemático de función: 


\section{$x=2 \quad f(x)=x 2 \quad f(2)=4$ \\ Forma' función Forma"}

Sea " $x$ " forma en función de determinada variable. Ejemplo: Piezas de metal ( $F$ ') se transforman en techo (F") en función de protegerse de la lluvia (utilitas), con estabilidad por bajo peso (firmitas) y factibilidad por bajo costo (sumptus); pero se podría transformar en techo de palma (F'") en función dar imagen rústica (significatio), reducción de ruido de lluvia y beneficiar salud (utilitas). Y así las distintas variables formales-materiales generarán performances funcionales distintas. Es una función técnica transformadora, pues consiste en el pasaje, mediante un dispositivo apropiado (criterio cualitativo), de un conjunto dado de estados iniciales de un sistema al conjunto deseado de estados finales. Esta sería la función relacional que menciona Abbagnano [17, p.516].

Una vez construido, materializado por la praxis tecnológica, el objeto pasa a un estado de función potencial mientras no sea usado. En el momento práctico, aparece la función operativa o "activación operativa de las cosas, para disfrutarlas plenamente en su uso" [21]. comprobándose entonces la función proyectiva, verificándose empíricamente si se satisfacen las necesidades o deseos humanos que la motivaron, donde se verifican las cualidades funcionales del objeto, donde se comprueba en la praxis la hipótesis de diseño. Es la perfomance donde el objeto cumple o no verdaderamente su finalidad.

\section{Devenir de la tríada forma-función-estructura}

En algún momento de la historia de la teoría arquitectónica, y del diseño en general, se introdujo la tríada de forma-función-estructura como categorías analíticas para analizar los objetos diseñados. Si vemos esos conceptos tal cual los entendemos en este artículo tenemos que: La forma es la estructura organizadora de la materia y el espacio, el modo como se organiza. La función es la activación operativa de los objetos en su uso, según su finalidad, y tiene su etapa proyectiva. La estructura es el conjunto de relaciones complejas que vemos dentro y entre todos los sistemas involucrados, es decir, entre el objeto y su contexto, y entre los contextos entre sí.

Esta tríada analítica tiene desarrollo en Marx referida a temas económicos. En "El Capital" la utiliza, en donde la materia y el trabajo humano producen objetos, y su valor como mercancía puede transformarse en diversas formas, como el oro o el dinero, formas distintas de valor, las cuales cumplen una función similar como forma común útil para el intercambio por otras mercancías. El concepto de estructura se aplica a las relaciones sociales y económicas, a las relaciones internas fundamentales de estos subsistemas del gran sistema económico, flujos de materia, energía (trabajo) o información (ideológicos).

"Marx, al suponer que la estructura no se confunde con las relaciones visibles sino que explica su lógica recóndita, anuncia la corriente estructuralista moderna... Así, método dialéctico y método estructural tendrían ante todo en común que ambos distinguen radicalmente las apariencias empíricas y las estructuras internas recónditas, que ambos conciben las apariencias empíricas como un modo de aparición, ordinariamente engañador, de las estructuras internas..." [22]. Es decir, las formas son la apariencia empírica de una estructura. Un ejemplo de Nils Castro [16, p.70): el patrón estructural del esclavismo puede adoptar distintas variantes según las sociedades, como serían las formas azteca o egipcia.

¿Cuándo se introduce la trilogía conceptual de forma-función-estructura en la teoría del diseño? No está claro. Henri Lefebvre, que escribió un ensayo titulado "Forma, función y estructura en El Capital", puede ser una pista, y en extenso libro "La producción del espacio" las pisadas a 
seguir. No llega a definir explícitamente los términos forma, función y estructura, a menudo es más retórico que preciso, pero en algunos renglones es posible entender algo de su interpretación y uso [9, p.198]: "Como toda realidad, el espacio social se relaciona metodológica y teóricamente con tres conceptos generales: forma, estructura, función. Es decir, cualquier espacio social puede devenir objeto de un análisis formal, estructural o funcional". Hay referencia a la forma geométrica, y a la exclusión de cualquier valoración estética en su descripción, tal como se ha denunciado aquí la contaminación estética del concepto de forma. Mención de la forma geométrica urbana y sus funciones ajustadas a la estructura económico-social del contexto. Estrecha correlación entre forma y estructura, como cuando se indicó en este artículo que la forma es una estructura y toda estructura tiene una forma. La centralidad como combinación de formas geométricas y topológicas definiendo una estructura espacial social y mental, para ciertas funciones [9, p.430]. La estructura también se concibe como la relación entre formas, y como la relación entre forma y función en una identidad denotable.

Pero en el capítulo XIV, Lefebvre derrapa [9, p.185], pues reduce el análisis estructural (estructuralista) a un problema de la estabilidad de la masa material y las relaciones entre las fuerzas físicas a las que son sometidas, es decir, a la estructura física soportante del objeto. Esta confusión conceptual entre estructura sistémica organizativa, como sistema de relaciones, respecto a la estructura soportante, como componente físico del objeto, ya se había mencionado al hablar de la forma y su estructura. Probablemente debido a que son dos palabras homónimas. Lo grave es que esta confusión se instaló dentro de la conceptualización de la tríada analítica forma-función-estructura aplicada a la arquitectura y, al parecer, un culpable sería Pier Luigi Nervi. No ha sido posible encontrar la fuente directa donde Nervi se refiere a la forma-función-estructura. Pero Philippe Boudon [4], donde habla de las "partes de la arquitectura", muestra una tabla de equivalencias (figura 6) a la tríada vitruviana utilitas-firmitasvenustas, donde referencia a Nervi.

\begin{tabular}{|c|c|c|c|}
\hline ALBERTI & voluptas & firmitas & commoditas \\
\hline BLONDEL & gracia & solidez & comodidad \\
\hline BLONDEL & decoración & construcción & distribución \\
\hline VTTRUVIO & venustas & firmitas & utilitas \\
\hline Soc. Cent. Arq. & belleza & verdad & útil \\
\hline GUIMARD & sentimiento & lógica & armonía \\
\hline NERVI & forma & estructura & función \\
\hline
\end{tabular}

Figura 6. Analogías conceptuales sobre "las partes de la arquitectura". Fuente: [4, p.20]

Igualmente, Arnau [23] señala que "cuando Nervi traduce a términos modernos la tríada cualitativa vitruviana utilitas-firmitas-venustas, su lectura es: función, estructura y forma. La cualidad firmitas se substancia y hace sustantivo en la estructura (soportante). Si bien es cierto que la solidez del edificio atañe a todas y a cada unas de sus partes, la estructura soportante es fundamental. La estructura es el sub-stantem, lo que está debajo de la edificación: su soporte".

Lo que ocurre es que Nervi confunde el objeto y sus cualidades, el sustantivo con el adjetivo, perdiéndose claridad conceptual: Equivale la estructura soportante, parte física de los objetos, con la cualidad de firmeza o estabilidad, propia de cualquier elemento material. Además, la estructura (soportante) queda aislada de la forma, cuando ella misma tiene una forma geométrica, y una forma topológica la organización de sus partes. Además, confunde la forma, 
que sería una característica morfológica del objeto, objetiva y denotable, con la cualidad de belleza, reincidencia de la contaminación estética del concepto de forma: la aconfusión de la 'species' con la 'venustas' de Vitruvio. Y para rematar, reduce el concepto de función solo al de utilidad práctica, dejando de lado las funciones semióticas (estéticas, simbólicas, emocionales, éticas). Esa equivalencia entre la venustas-firmitas-utilitas con la tríada forma-estructura-función es un desastre epistemológico. A partir de aquí cayeron en cascada miles de textos confusos.

\section{Conclusión}

Ha existido ambigüedad en el concepto de forma, además de sufrir una contaminación de valoración estética; se denuncia esta corrupción, se enfatiza como categoría exclusivamente morfológica, y se proponen como suficientes la forma topológica y forma geométrica, que tienen su aplicación secuencial durante el diseño. El concepto de función, tradicionalmente aplicado a la utilidad práctica de los objetos, se amplía hacia su función semiótica, como parte de la teleología de los objetos. Se distingue entre función proyectiva y operativa, morfogenética una y de activación práctica la otra, correspondientes a los estadios de diseño y uso de los objetos. Al concepto de estructura, que en ciertos casos ha sufrido una deformación conceptual al reducirse a la estructura física soportante de los objetos, se le restituye su sentido sistémico relacional, distinguiéndose las estructuras intrínsecas formales y materiales del objeto, y las hiperestructuras de relaciones externas (o extructuras, como neologismo propio) como son las correlaciones funcionales entre objeto y usuario, o las vínculos contextuales entre el objeto y su entorno.

Para el ámbito que nos concierne, el análisis basado en estas categorías generales debe aplicarse, obviamente, sobre los objetos diseñados, el entorno humano que los demanda, y entorno natural y construido que los circunda. Son necesarias otras categorías particulares, como la definición de objeto -o forma materializada funcional artificial- y su hiperestructura de cualidades, que tienen que ver con su ajuste o adaptación con el usuario y su entorno: Objeto - cualidades - contexto. La descripción de las cualidades funcionales a nivel semiótico y de utilidad práctica, y su estabilidad, costo económico y ambiental, es materia de otro ensayo.

\section{Referencias}

[1] W. Tatarkiewicz, Historia de seis ideas. Madrid: Tecnos, 2001.

[2] J. Ferrater Mora, Diccionario de Filosofía de Bolsillo 1. Madrid: Alianza, 1997, p.371.

[3] M. Bunge, Diccionario de filosofía. México D.F.: Siglo XXI, 2005, p.87.

[4] Ph. Boudon, Del espacio arquitectónico. Buenos Aires: Victor Lerú, 1980.

[5] Ch. Alexander, "La ciudad no es un árbol", Ciudades para un futuro más sostenible, Boletín CF+S 40, Junio 2009. [En línea] Disponible en: http://habitat.aq.upm.es/boletin/n40/

[6] A. Millán Gómez, Aproximación a una taxonomía topológica de formas arquitectónicas y urbanas. Universitat Politècnica de Catalunya, 1981. Barcelona: Tesis Doctorales en Red. [En línea] Disponible en: http://www. tesisenred.net/handle/10803/6096

[7] I. Blanco y M. de Prada, Topología y arquitectura. Verdades aparentes y realidades estructurales. Artículo independiente. Madrid: Universidad Politécnica de Madrid, 2016. [En línea] Disponible en: http://oa.upm. es/39227/1/TFG_Ivan_Blanco_Longa.pdf

[8] Vitruvvi Pollionis, M, De Architectvra. Venecia: Franciscus Franciscium y loan Crugher, 1507, Liber III, Caput III, p.103. [En línea] Disponible en: https://arts.st-andrews.ac.uk/digitalhumanities/fedora/repository/ digitalcollections\%3A606\#page/1/mode/2up, o en: https://reader.digitale-sammlungen.de/de/fs1/object/display/bsb10195988_00011.html

[9] H. Lefebvre, La producción del espacio. Madrid: Capitán Swing, 2013.

[10] Ch. Norberg-Schulz, Intenciones en arquitectura. Barcelona: Gustavo Gili, 1988, p.85. 
[11] E. Tedeschi, Teoría de la arquitectura. Buenos Aires: Nueva Visión, 1972, p.206.

[12] A. Borie, P. Micheloni, P. Pinon, Forma y deformación. Barcelona: Reverté, 2008.

[13] P. Collins, Los ideales de la arquitectura moderna (1750-1950). Barcelona: Gustavo Gili, 2001, pp.162 y 223.

[14] M. M. Rosental y G. M. Straks, Categorías del materialismo dialéctico. México D.F.: Grijalbo, 1960, p.197.

[15] M. Arnold, Marcelo y F. Osorio, "Introducción a los Conceptos Básicos de la Teoría General de Sistemas", en Cinta moebio 3: 40-49. Santiago: Universidad de Chile, 1998, sin página. [En línea] Disponible en: http://www. facso.uchile.cl/publicaciones/moebio/03/frprinci.htm

[16] H. Lefebvre, A. Sánchez Vásquez, et al, Estructuralismo y marxismo. México D.F.: Grijalbo, 1970.

[17] N. Abbagnano, Diccionario de filosofía. México D.F.: Fondo de Cultura Económica, 2007.

[18] B. Malinowski, Una teoría científica de la cultura. Barcelona: Edhasa, 1970.

[19] A. Maslow, Motivación y personalidad. Madrid: Días de Santos, 1991, p.21-33.

[20] Ch. Alexander, Ensayo sobre la síntesis de la forma. Buenos Aires: Infinito, 1976.

[21] J. R. Morales, Arquitectónica. Madrid: Biblioteca Nueva, 1999, p.119.

[22] E. Trías et al, Estructuralismo y marxismo. Barcelona: Martínez Roca, 1969, p.117.

[23] J. Arnau, 72 voces para un diccionario de arquitectura teórica. Madrid: Celeste, 2000, p.78. 